\title{
Thyroid Gland Capsule
}

National Cancer Institute

\section{Source}

National Cancer Institute. Thyroid Gland Capsule. NCI Thesaurus. Code C33781.

The fibrous lining that surrounds the left and right lobes of the thyroid gland. 